m/s

médecine/sciences $1996 ; 12: 703-5$

\section{LE CERVEAU HUMAIN : UN ORDINATEUR À RÉSEAUX MULTIPLES ET FRAGILES...}

\section{Daniel Widlöcher}

\section{ADRESSE}

D. Widlöcher : professeur à l'université Pierre-etMarie-Curie, médecin du groupe Hospitalier PitiéSalpêtrière, 47-83, boulevard de l'Hôpital, 75651 Paris Cedex 13, France. a pathologie liée à des atteintes lésionnelles ou fonctionnelles du cerveau va connaître des développements nouveaux dans un très proche avenir grâce à deux champs d'investigation, l'imagerie cérébrale d'une part et la génétique de l'autre. Les domaines qui donnent matière à une partie importante des articles de synthèse de ce numéro en constituent une bonne illustration. Ils montrent que ces développements peuvent être attendus dans l'étude et le traitement tant des processus maturatifs de l'enfance que des processus dégénératifs de la sénescence.

Les techniques d'imagerie cérébrale permettent de localiser des anomalies fonctionnelles ou lésionnelles. Elles reposent, on le sait, sur deux méthodes principales. La première se fonde sur l'étude, par calcul de données informatisées, de radiations émanant de produits radio-actifs introduits dans l'organisme [1]. La tomographie par émission de positons (ou positrons) (PET-scan) utilise différents radio-isotopes (en rapport avec le métabolisme du glucose, la consommation d'oxygène et les sites de localisation de médicaments marqués) ( $m / s n^{\circ} 4$, vol. 7, p. 393). Dans les études fonctionnelles, l'indicateur utilisé est le flux sanguin. Déjà en 1890, Sherrington avait suggéré l'existence d'un «mécanisme auto- matique » qui règle le flux sanguin dans le cerveau en fonction des variations locales de l'activité fonctionnelle. Le PET scan est en effet un outil commode et très sûr pour mesurer le flux sanguin cérébral à partir d'un oxygène marqué. On a pu ainsi mesurer des variations de flux très rapides avec une bonne résolution spatiale, confirmant ainsi l'hypothèse de Sherrington. Un autre dispositif s'inspire des mêmes principes : le SPECT (single photon emission ccomputerized tomography) utilise des ligands radio-actifs qui se fixent sur les membranes endothéliales [2]. La relative lenteur d'élimination permet une plus grande souplesse et donne à cette technique certains avantages, en particulier de coût, qui en font une épreuve intéressante dans les épreuves fonctionnelles.

La seconde méthode, fondée sur la résonance magnétique nucléaire, utilise la capacité de nombreuses structures atomiques soumises à un champ magnétique de se comporter comme un indicateur magnétique [3]. On peut ainsi recueillir les déplacements d'orientation de protons et cette détection permet d'étudier la charge en oxygène de l'hémoglobine, qui s'est révélée ainsi un indicateur sensible de l'activité neuronale. L'intérêt des techniques d'imagerie par résonance magnétique est l'absence de traceurs à injecter et la très bonne résolution 
spatiale. Leur développement a constitué un progrès spectaculaire dans le champ de l'exploration in vivo de l'activité cérébrale.

Comme le montre l'étude des mécanismes cérébraux de la lecture (voir l'article de M. Habib et F. Robichon, p. 707 de ce numéro) on assiste ainsi au renouvellement des recherches sur les localisations fonctionnelles que la méthode anatomo-pathologique avait permises depuis les travaux de la fin du siècle dernier. On notera toutefois une différence : la méthode anatomo-pathologique cherchait à définir des centres que l'on mettait en rapport avec les observations cliniques [4]. Ici le modèle est différent. On peut se représenter les aires cérébrales comme constituant un réseau complexe et échangeant entre elles des informations, la technique d'imagerie permettant d'observer comment fonctionne le réseau. Elle se rapproche ainsi des enregistrements électro-encéphalographiques. Elle demeure encore actuellement moins sensible que ces derniers à la dimension temporelle mais beaucoup plus riche en informations quant aux localisations spatiales. Le développement de systèmes d'exploitation de techniques complémentaires entre ces différentes méthodes permettra de nombreux progrès.

L'application des sciences cognitives à la neuropsychologie a modifié la référence à la clinique. Il ne s'agit plus d'observer des symptômes mais d'étudier les mécanismes du traitement de l'information et de la réalisation des opérations mentales qui concourent à la production du déficit. Il ne s'agit donc plus d'une neurologie fondée sur la comparaison entre des symptômes cliniques et une lésion mais d'une neuropsychologie qui met en rapport des opérations mentales très élémentaires avec des altérations fonctionnelles des circuits neuronaux. La technique actuellement la plus utilisée s'appuie sur des opérations de soustraction, bien illustrées dans l'article de M. Habib et F. Robichon consacré à la lecture (p. 707 de ce numéro). Cette technique consiste à comparer une épreuve cognitive simple avec une épreuve cognitive plus complexe et de sous- seconde situation celles des zones activées par la première.

Il ne faut pas toutefois se dissimuler les difficultés méthodologiques qui rendent complexe l'interprétation des résultats. Par ailleurs, la localisation de zones cérébrales affectées par le processus ne signifie pas nécessairement l'existence d'une lésion causale. La finesse des analyses permet en effet d'observer des défauts d'activation ou des activations nouvelles en fonction de la complexité de l'épreuve cognitive, sans que l'anomalie constatée soit nécessairement la cause du trouble. Elle peut en effet refléter simplement le dérèglement fonctionnel qui peut être dû à des événements extérieurs, des stimuli ou des conditionnements pathogènes. Néanmoins, l'approche cognitive permet déjà des techniques rééducatrices nouvelles dans les domaines qui concernent des populations importantes d'enfants. Ces progrès ne doivent pas faire oublier non plus les retentissements motivationnels et émotionnels liés au trouble. En effet, on peut obtenir dans un très grand nombre de protocoles des anomalies détectables par ces techniques d'imagerie chez des sujets témoins en fonction de la complexité de la tâche, du stress émotionnel créé, etc.

Cette dernière réserve s'applique également à l'autisme infantile (voir l'article de G. Lelord et al., p. 715 de ce numéro). Les premiers résultats obtenus par l'imagerie cérébrale dans ce domaine permettent d'identifier des zones impliquées dans la production des symptômes. Les études électrophysiologiques et d'imagerie fonctionnelle ont montré des anomalies de l'activité cérébrale que l'on peut mettre en parallèle avec certaines anomalies de l'activité sensori-motrice et cognitive. On a ainsi mis en évidence des troubles de la réactivité de l'hémisphère gauche à des stimulations auditives qui laissent penser qu'il existe une perturbation sévère du fonctionnement cortical. Toutefois, dans l'état actuel de nos connaissances, il est difficile de préciser ce qui serait dû à un trouble du développement ou à une simple anomalie fonctionnelle en réponse à des facteurs liés à l'environnement. Par ailleurs, l'individualisation de mécanismes génétiques permet d'envisager une meilleure connaissance de la physiopathologie de l'autisme et, peut-être, une intervention chimiothérapique sélective. Ici encore, si la suspicion forte d'un processus anormal au cours du développement conduit à mettre en œuvre des techniques de rééducation, cette orientation n'invalide pas la nécessité de prendre en compte les aspects émotionnels et de l'ordre de la motivation. Récemment, le Comité Consultatif National d'Éthique pour les Sciences de la Vie et de la Santé a été saisi d'une plainte formulée par des parents d'enfants autistes concernant l'insuffisance des recherches scientifiques dans ce domaine, le caractère arbitraire des choix thérapeutiques et l'absence d'évaluation des résultats. Sans entrer dans un débat passionné et souvent marqué par des a priori théoriques, on peut dire que des travaux comme ceux présentés ci-dessous montrent que les recherches se développent. En matière de traitement, dans l'ignorance où nous sommes des causes et des mécanismes, il faut adopter une position éclectique. Il est impossible actuellement de s'en tenir à une stratégie thérapeutique univoque (médicaments, psychothérapies relationnelles, techniques éducatives) : cliniciens et familles doivent s'entendre en disposant de toutes les informations nécessaires. Il serait, en revanche, hautement souhaitable que l'évaluation des soins soit l'objet de recherches méthodiques.

Les travaux génétiques actuels, comme nous le voyons à propos de l'autisme infantile, orientent vers une explication multigénique de ces maladies. On retrouve d'ailleurs cette perspective dans l'étiologie de la maladie d'Alzheimer (voir l'article de D. Campion et al., p. 723 de ce numéro). Ici cependant, si les travaux de génétique moléculaire peuvent nous permettre de mieux comprendre le mécanisme, il ne faut pas méconnaître la dimension éthique du problème. Chaque fois en effet que nous progressons dans l'étude des facteurs de risque génétique, nous devons nous interroger sur les conséquences psychologiques des informations ainsi communiquées aux familles. Dans le cas de l'autisme, ces informations 
ont quelque utilité, en particulier pour permettre un diagnostic précoce et des mesures psychologiques appropriées. Dans le cas de la maladie d'Alzheimer, la situation est toute différente. Nous sommes probablement encore assez loin du moment où l'on pourra isoler des sousgroupes déterminés par des mécanismes moléculaires précis pour envisager des traitements spécifiques. Il est permis toutefois d'attendre des résultats très significatifs des nouvelles méthodes d'investigation

\section{RÉFÉRENCES}

1. Posner MI, Petersen SE, Fox PT, Raichle ME. Localization of cognitive functions in the brain. Science 1988 ; 240 : 1627-31.

2. Risberg J. Regional cerebral blood flow measurements by 133 Xe-inhalation : methodology and applications in neuropsychology and psychiatry. Brain Lang 1980 ; 9 : 9-34.

3. Grenier, P, Desbleds M, Guilbeau J. L'imagerie par résonance magnétique se substituera-t-elle à la tomodensitometrie ? médecine/sciences 1986 ; 2 : 130-6.

4. Déjerine J. Contribution à l'étude anatomo-clinique et clinique des différentes variétés de cécité verbale. Mém Soc Biol 1892 ; 4 : 61-90. 